\title{
Qualitative analysis of Apis mellifera L. honey in an ecotone area in the Bahian semiarid
}

Análise qualitativa do mel de Apis mellifera L. em uma área de ecótono no semiárido baiano

\author{
H. S. Reis ${ }^{1,2}$; S. O. Araújo ${ }^{2}$; L. C. L. Lima ${ }^{1}$; F. H. M. Silva ${ }^{1,2 *}$ I. J. S. Diogo ${ }^{3}$; M. \\ D. Saba ${ }^{1,2}$ \\ ${ }^{1}$ Programa de Pós-graduação em Biodiversidade Vegetal, Universidade do Estado da Bahia, 48970-000, Senhor do \\ Bonfim-BA, Brasil \\ ${ }^{2}$ Laboratório de Estudos Palinológicos, Universidade do Estado da Bahia, 48970-000, Senhor do Bonfim-BA, Brasil \\ ${ }^{3}$ Coordenação do Curso de Ciências Biológicas, Instituto Federal da Paraíba, 58755-000, Princesa Isabel-PA, Brasil
}

*hildermagalhaes@hotmail.com

(Recebido em 04 de novembro de 2020; aceito em 20 de maio de 2021)

\begin{abstract}
This study aimed to identify the pollen types of 11 honey samples produced in apiaries of two cities in the State of Bahia, Brazil: Antônio Gonçalves (five samples) and Campo Formoso (six samples); to verify richness and diversity from each apiary and to compare the similarity among them. The samples were obtained from beekeepers according to the period of production and availability. The palynological analysis of these samples followed the standard procedures of melissopalynology with the use of acetolysis. The pollen types occurring in the honey samples were identified and at least 1,000 pollen grains per sample were counted to determine the frequency classes and the frequency of occurrence. Overall, 147 pollen types were detected, of which 143 are distributed in 35 botanical families. The most representative families concerning the number of pollen types were: Fabaceae (33), Asteraceae (18), Euphorbiaceae (11), Anacardiaceae and Malvaceae (09 types each), Rubiaceae (08) and Myrtaceae (07). The average value of pollen types per sample was 28.18. The genus that contributed the most was Mimosa L. (Fabaceae) with eight pollen types detected. The wealth of pollen types found revealed important information about the local flora which is explored by Apis mellifera. Therefore, it becomes an important tool to assist in the preservation and propagation of these plants and, consequently, increasing honey production.
\end{abstract}

Keywords: bee product, botanical origin, palynology.

Este trabalho teve como objetivo identificar os tipos polínicos de 11 amostras de mel produzidas em apiários de duas cidades baianas: Antônio Gonçalves (cinco amostras) e Campo Formoso (seis amostras); verificar a riqueza e diversidade de cada apiário e comparar a similaridade entre eles. As amostras foram obtidas junto aos apicultores de acordo com o período de produção e disponibilidade. A análise palinológica dessas amostras seguiu o procedimento padrão da melissopalinologia com o uso da acetólise. Os tipos polínicos que ocorreram nas amostras de mel foram identificados e pelo menos 1.000 grãos de pólen por amostra foram contados para determinar as classes de frequência e frequência de ocorrência. Foram detectados 147 tipos polínicos, dos quais 143 estão distribuídos em 35 famílias botânicas. As famílias mais representativas quanto ao número de tipos polínicos foram: Fabaceae (33), Asteraceae (18), Euphorbiaceae (11), Anacardiaceae e Malvaceae (09 tipos cada), Rubiaceae (08) e Myrtaceae (07). O valor médio dos tipos polínicos por amostra foi 28,18 . O gênero que mais contribuiu foi Mimosa L. (Fabaceae) com oito tipos polínicos detectados. A riqueza de tipos polínicos encontrados revelou informações importantes sobre a flora local que é explorada por Apis mellifera. Portanto, torna-se uma importante ferramenta para auxiliar na preservação e propagação dessas plantas e, consequentemente, no aumento da produção de mel.

Palavras-chave: produtos apícolas, origem botânica, palinologia.

\section{INTRODUCTION}

Beekeeping has an essential ecological characteristic, which allows the regional conservation of bee species and honey-producing plants. It is an economic activity of increasing importance in family production systems and of easy maintenance, being, therefore, determinant in the improvement of life quality and permanence of the man in the rural environment [1-5]. Among the main products obtained and commercialized by beekeeping, honey, bee pollen, propolis, royal jelly, wax and apitoxin stand out, products of the hive with a wide range of nutraceutical applications [5, $6]$. 
Honey consists of balanced portions of glucose, vitamins, minerals, acids and amino acids. It is one of the easiest bee products to be explored, either as a food source or as an input in the pharmaceutical and cosmetic industries [6,7]. In Brazil, most of the production depends on native forests to obtain nectar, pollen, and resin. Thus, beekeeping plays an important role in the environment, intensifying the process of pollination of plant crops and, consequently, the necessary fertilization for fruiting $[1,8,9]$.

Bees act as natural pollinators of different plant species, contributing to the balance of the ecosystem and preservation of biodiversity [6]. Therefore, considering the foraging behavior of the bee species Apis mellifera L. 1958 (which is broad and with no food preference for specific groups of plants), the analysis of the pollen content in the honey produced by the colonies may reveal components of the flora of different environments. Since it is a natural hybrid, this species is considered quite productive and docile, when compared to bees of African and European origin, respectively $[10,11]$.

When collecting the nectar from the flowers, the bees also end up transporting the pollen grains, which are regurgitated together with the nectar in the honeycombs. The pollen grains of nectariferous, poliniferous and/or nectariferous-poliniferous plants are part of the honey. Therefore, the palynological analysis of this product is considered reference for the characterization of honeys in terms of botanical, regional and temporal origin [12-14]. This kind of knowledge is extremely important to beekeepers and specialized institutions for indicating adequate and abundant supply of nectar and pollen, to contribute to the preservation of plant species, and to increase the regional productivity $[15,16]$.

In the northeast region of Brazil, studies on this subject, such as those by Moreti et al. (2000) [17], Moreti et al. (2005) [18], Sodré et al. (2007) [19], Sodré et al. (2008) [20], Dórea et al. (2010) [21], Oliveira et al. (2010) [22], Costa et al. (2015) [23], Nascimento et al. (2015) [24] and Santos et al. (2019) [25], contributed to the recognition of the botanical affinity of honeys and pollen loads collected by A. mellifera. Oliveira and Santos (2014) [11] and Santos et al. (2020) [26] performed the palynological analysis of A. mellifera honey samples from cities located in the State of Bahia. For the identification of pollen types found in these honeys, a thorough analysis is necessary and requires specific knowledge of palynology and the local flora [27, 28].

The cities of Antônio Gonçalves and Campo Formoso, located in the semiarid region of Bahia, Brazil, have invested in increasing honey production in recent years, however the systematic palynological studies of these products are still incipient and the available knowledge is limited, covering few areas of the State. For this reason, this study aimed to identify and botanically characterize the types of pollen in A. mellifera honey from apiaries located in these cities, analyzing richness, diversity and similarity to expand knowledge about the botanical species most exploited by bees in the search for their trophic resources, mainly for honey production.

\section{MATERIALS AND METHODS}

\subsection{Study area}

The cities of Antônio Gonçalves (10²'22”'S; 40¹6’31"W; 472 a.s.l) and Campo Formoso (10 $30^{\prime} 27^{\prime \prime} S ; 40^{\circ} 19^{\prime} 17^{\prime \prime} \mathrm{W} ; 556$ a.s.l.) are located in the north-central region of the State of Bahia, Brazil (Figure 1). These cities are approximately $9.0 \mathrm{~km}$ apart and are part of a group of nine cities comprising the Território Piemonte Norte do Itapirucu [29].

The climate varies from sub-humid to dry in Antônio Gonçalves, and semiarid in Campo Formoso, with average annual temperatures varying between $16^{\circ} \mathrm{C}$ to $29^{\circ} \mathrm{C}$. The regional vegetation is the typically rarefied arboreal Caatinga, which is adapted to the local rainfall regime that varies from 300 to $1,100 \mathrm{~mm} /$ year, with the rainy season in March and a relatively dry season in September. Antônio Gonçalves is characterized by Caatinga-Floresta Estacional and CerradoCaatinga and Campo Formoso by an open and dense arboreal Caatinga, besides relictuals of the Atlantic forest [29]. 


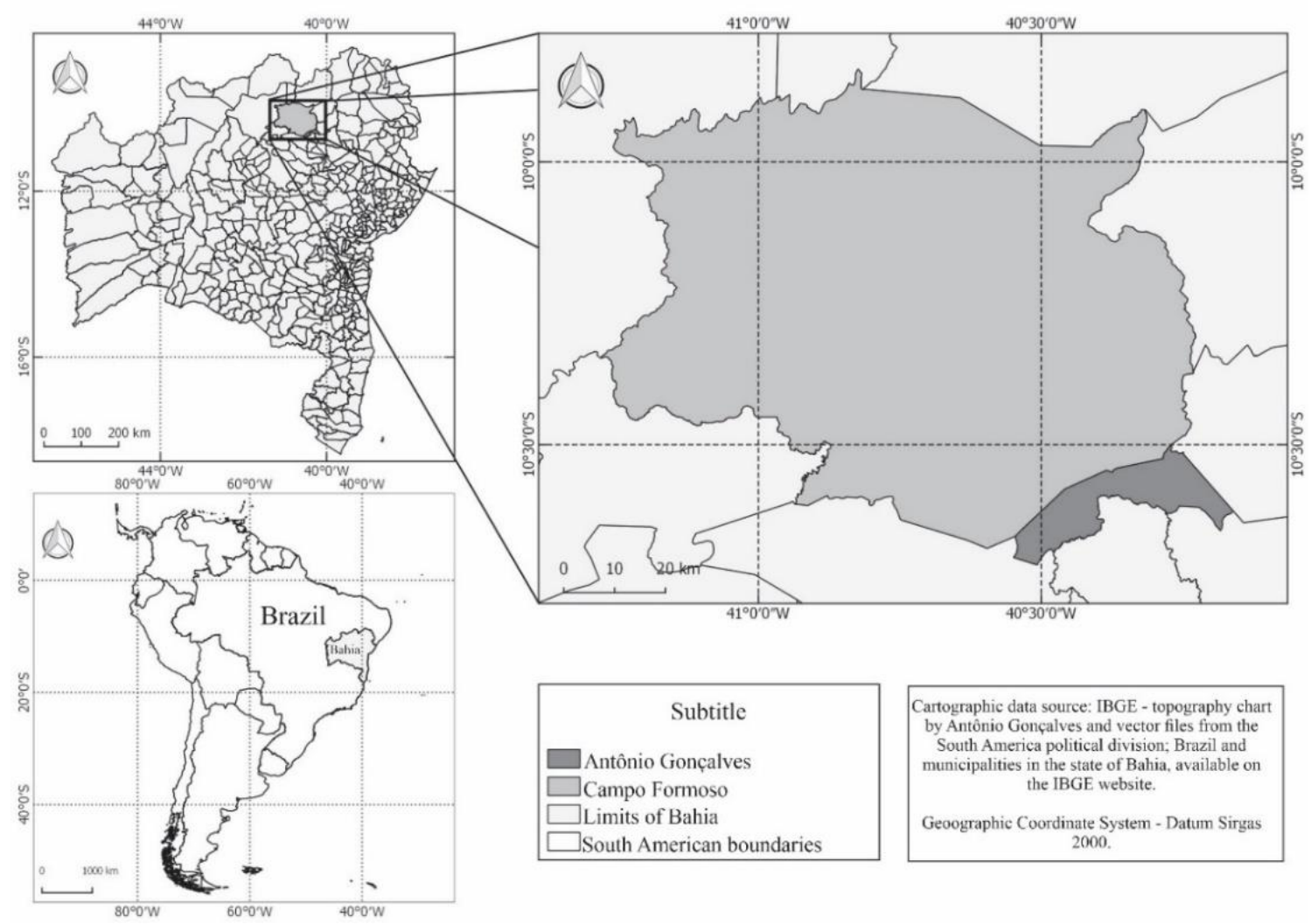

Figure 1: Location map of the cities of Antônio Gonçalves and Campo Formoso, Bahia, Brazil.

\subsection{Collection and processing of honey samples}

It was obtained $11 \mathrm{~A}$. mellifera honey samples from the Cooperativa Apícola e Pesqueira de Campo Formoso (COOAPCAF) that were produced in apiaries located in the cities of Campo Formoso [Roça da Fonte, Borda da Mata, Monteiro, Fazenda Tombão and Alagadiço do Brejão (samples I, II, III, IV, V VI)] and Antônio Gonçalves [Caldeirão do Mulato and Lagoa Grande (samples VII, VIII, IX, X, XI)], Bahia. The collection of samples followed the period of production, harvest and availability (Table 1).

Table 1: Collection period of honey samples (I - XI) from different apiaries located in Campo Formoso and Antônio Gonçalves, Bahia, Brazil.

\begin{tabular}{|c|c|c|c|c|c|c|c|c|}
\hline \multirow[b]{3}{*}{ Year } & \multirow[b]{3}{*}{ Months } & \multicolumn{7}{|c|}{ Apiaries } \\
\hline & & \multicolumn{5}{|c|}{ Campo Formoso } & \multicolumn{2}{|c|}{ Antônio Gonçalves } \\
\hline & & $\begin{array}{c}\text { Roça } \\
\text { da } \\
\text { Fonte }\end{array}$ & $\begin{array}{c}\text { Borda } \\
\text { da } \\
\text { Mata } \\
\end{array}$ & Monteiro & $\begin{array}{l}\text { Fazenda } \\
\text { Tombão }\end{array}$ & $\begin{array}{l}\text { Alagadiço } \\
\text { do Brejão }\end{array}$ & $\begin{array}{r}\text { Caldeirão } \\
\text { do Mulato }\end{array}$ & $\begin{array}{c}\text { Lagoa } \\
\text { Grande }\end{array}$ \\
\hline \multirow[t]{6}{*}{2014} & May & & II & & & & & \\
\hline & June & I & & & & & & \\
\hline & July & & & III & & & & \\
\hline & September & & & & V & & & \\
\hline & October & IV & & & & & VII & \\
\hline & November & & & & & VI & & \\
\hline \multirow[t]{2}{*}{2015} & August & & & & & & VIII & \\
\hline & December & & & & & & IX & \\
\hline 2016 & May & & & & & & $\mathrm{X}$ & XI \\
\hline
\end{tabular}


The processing and palynological analyses of the pollen content in the samples were made at the Laboratório de Estudos Palinológicos (LAEP) of the Universidade do Estado da Bahia - Campus VII. The standard technique of Louveaux et al. (1978) [30], adapted by Iwama and Melhem (1979) [31], was followed to process pollen grains present in the honey samples. The pollen sediment was acetolyzed according to the method of Erdtman (1960) [32]. The slides were mounted with glycerin jelly and analyzed under light microscopy, for counting and identifying pollen types.

To determine the percentages and frequency classes of the pollen types recorded in the honey samples, 1,000 pollen grains per sample were counted consecutively. Pollen types were grouped into four categories established by Louveaux et al. (1978) [30]: predominant pollen (PP) (more than $45 \%$ of the pollen grains in a sample); secondary pollen (SP) (16 to $45 \%$ of the sample); important minor pollen (IMP) (3 to 15\% of the sample); minor pollen (MP) (less than $3 \%$ of the sample).

The frequency of occurrence of the pollen type per sample set was also estimated, using the following equation: $\mathrm{FO}=(\mathrm{ni} / \mathrm{N}) \times 100(\mathrm{FO}=$ frequency of occurrence of the pollen type; $\mathrm{ni}=$ pollen type number counted in the sample; $\mathrm{N}=$ total number of pollen grains counted in the sample). According to Louveaux et al. (1978) [30], the pollen types were grouped into the following categories: very common (pollen types present in more than $45 \%$ of the total samples analyzed); frequent (present in 16 to $45 \%$ of samples); rare (present in 3 to $15 \%$ of samples), and sporadic (present in less than $3 \%$ of samples).

The pollen types were identified and classified based on comparisons with reference slides deposited in the pollen collection (Palinoteca) of LAEP/UNEB and the Laboratorio de Micromorfologia Vegetal of the Universidade Estadual de Feira de Santana (LAMIV/UEFS), which are composed mainly of taxa from the Caatinga's flora. Besides that, descriptions in literature, catalogs, and specialized articles were also consulted, such as Lima et al. (2006) [28], Lima et al. (2008) [33] and Buril et al. (2010) [34] (used for Mimosa spp.), Barth (1989) [12], Borges et al. (2006) [27], Silva et al. (2010) [35] and Silva et al. (2016) [36].

All pollen types found in the samples were outlined and the representative material illustrated through photomicrographs obtained from a photomicroscope (Zeiss, Axioscop 40) from LAEP/UNEB.

\subsection{Statistical analysis}

The Diversity Index of Shannon-Weiner $\left(\mathrm{H}^{\prime}\right)$ [37] and the Dominance index of Simpson (D) [38] were calculated to the pollen data in all the samples and in each apiary, according to the below equations in $\mathrm{R}$ program, vegan package [39]. Both indexes were performed because ShannonWeiner accounts for entropy in representative samples, whereas Simpson is used to estimate dominance.

$$
\mathrm{H}^{\prime}=-\Sigma \text { pi } \mathrm{x} \ln \mathrm{pi},
$$

where: $\mathrm{pi}=\mathrm{ni} / \mathrm{N}, \mathrm{ni}=$ number of individuals of the species $\mathrm{i}, \mathrm{N}=$ total number of sampled individuals.

$$
1-\mathrm{D}=1-\Sigma\{[\mathrm{ni}(\mathrm{ni}-1)] / \mathrm{N}(\mathrm{N}-1)\},
$$

where: $\mathrm{ni}=$ number of individuals of $\mathrm{n}$-th species, $\mathrm{N}=$ total number of sampled individuals.

The similarity analysis was conducted using the data of the samples from the two cities, considering the seven apiaries. The analysis was performed with the aid of the software PAST Palaeontological Statistics, Ver. 3.11 [40]. We carried out an unweighted pair group method with arithmetic mean (UPGMA) agglomerative cluster analysis with Jaccard's similarity coefficient applied, as it does not consider shared absences as evidence of similarity. For the identified pollen types, presence or absence data were considered. 


\section{RESULTS}

One hundred and forty-seven pollen types were found in honey samples from the cities of Antônio Gonçalves and Campo Formoso, Bahia. Of the total, 143 pollen types had their botanical affinity established, being distributed in 34 families (Figure 2; Table 2).

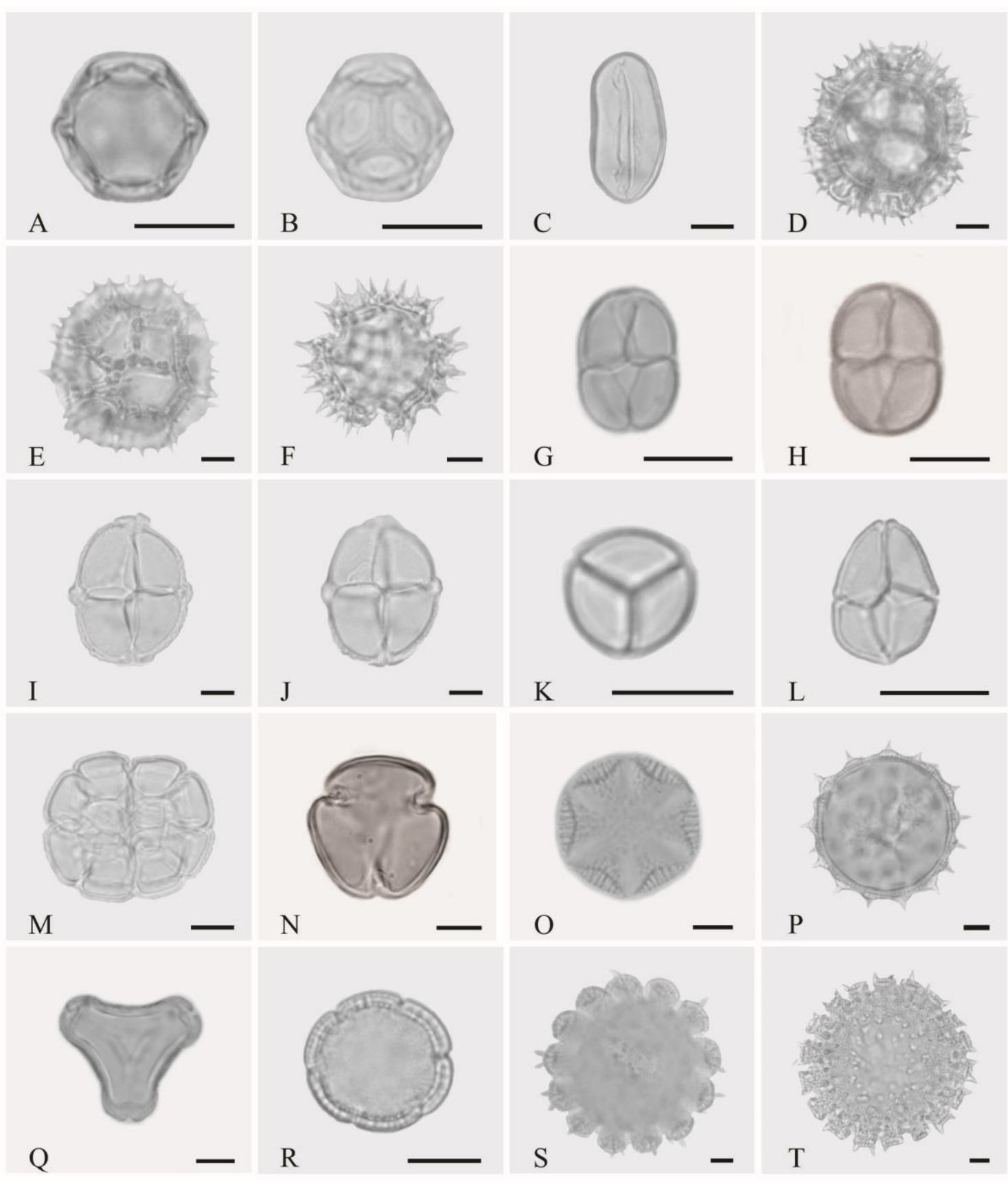

Figure 2: Some pollen types found in the cities of Antonio Gonçalves and Campo Formoso, Bahia, Brazil. Amaranthaceae: A-B-Alternanthera brasiliana. Arecaceae: $C$-Syagrus coronata. Asteraceae: D-ELepidaploa. F-Vernonia. Fabaceae: G-Mimosa arenosa. H-Mimosa caesalpiniifolia. I-J-Mimosa candolei. $K$ - Mimosa sensitiva. L-Mimosa tenuiflora. M-Senegalia bahiensis. $N$ - Prosopis. Lamiaceae: $O$-Salvia. Malvaceae: $P$-Herissantia. Myrtaceae: $Q-$ Eucalyptus. Rubiaceae: $R-$ Borreria verticillata. S-Diodia. T-Richardia grandiflora. (Scale 10 1 m).

Among the pollen types detected, only 63 were identified at the species level, and the rest were restricted only to the genus (70) and/or family (10). Four pollen types remained undetermined. The unidentified pollen types appeared at a low frequency, with $7.6 \%$ the highest percentage obtained 
in sample I. In sample II, indeterminate types 03 and 04 were classified as minor pollen $(<3 \%)$, as well as type 04 in sample III (Table 2).

The families Fabaceae, Asteraceae, Anacardiaceae and Malvaceae contributed significantly to the composition of the honey and presented pollen types in all analyzed samples. Pollen types of Rubiaceae were found in $90.90 \%$ of the samples, followed by Myrtaceae and Euphorbiaceae in 81.81\%, Poaceae in $72.72 \%$, and Amaranthaceae, Arecaceae and Lamiaceae in $63.63 \%$.

The contribution of pollen types for each botanical family was variable. Fabaceae was the most representative family with 33 identified pollen types, followed by Asteraceae (18), Euphorbiaceae (11), Anacardiaceae and Malvaceae (9 each), Rubiaceae (8), Myrtaceae (6), Poaceae (5), Arecaceae and Convolvulaceae (4). There were 18 contributing families according to the pollen spectrum of honey in the study region (Figure 3).

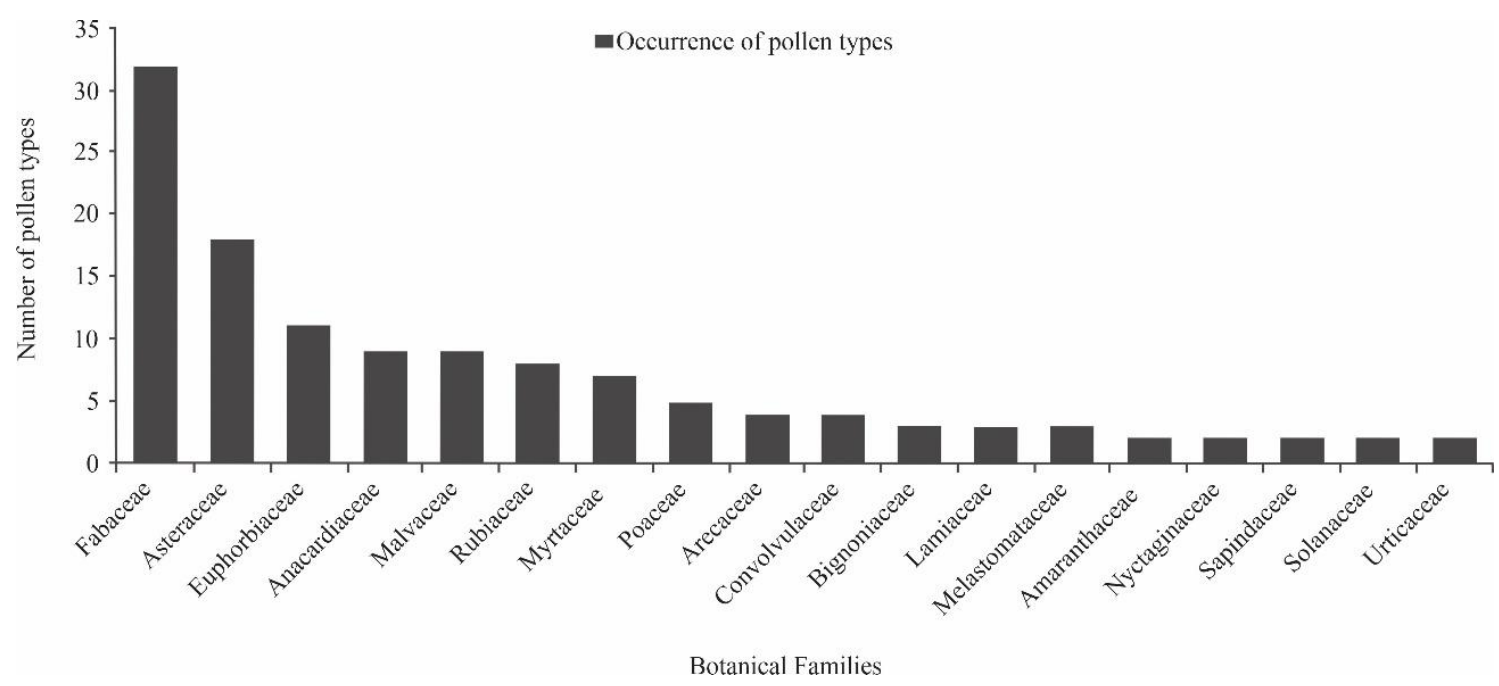

Figure 3: Main botanical families in number of pollen types identified in the honey samples analyzed from the cities of Antônio Gonçalves and Campo Formoso, Bahia, Brazil.

The genus Mimosa L. (Fabaceae) predominated in the number of pollen types in honey samples from both cities, being represented by eight species and a small number of grains not attributable to species, and identified as Mimosa sp.: M. arenosa, M. caesalpiniifolia, M. candollei, M. sensitiva, M. setosa, M. setuligera, M. tenuiflora, and M. verrucosa. Among the samples analyzed, most pollen types from this genus occurred mostly as important minor pollen (3-15\%) and minor pollen (Table 2).

Table 2: Pollen types present in honey samples analyzed in a semiarid area in Bahia, Brazil. Frequency of occurrence (FO): VF-very frequent (>45\%), $F$ - frequent (16-45\%) and $R$ - rare (3-15\%). Frequency classes: $P P$ - predominant pollen (>45\%), SP - secondary pollen (16-45\%), IMP - important minor pollen (3-15\%) and MP - minor pollen (<3\%).

\begin{tabular}{|c|c|c|c|c|c|c|c|c|c|c|c|c|}
\hline \multirow{2}{*}{ Pollen type } & \multirow{2}{*}{ FO } & \multicolumn{11}{|c|}{ Honey samples } \\
\hline & & I & II & III & IV & $\mathbf{V}$ & VI & VII & VIII & IX & $\mathbf{X}$ & XI \\
\hline Acanthaceae & & & & & & & & & & & & \\
\hline $\begin{array}{l}\text { Ruellia } \\
\text { Amaranthaceae }\end{array}$ & $\mathrm{R}$ & & MP & & & & & & & & & \\
\hline $\begin{array}{l}\text { Alternanthera } \\
\text { brasiliana }\end{array}$ & VF & MP & & & & MP & IMP & MP & IMP & MP & MP & \\
\hline Gomphrena & $\mathrm{R}$ & & & & & & MP & & & & & \\
\hline Anacardiaceae & & & & & & & & & & & & \\
\hline Anacardiaceae I & $\mathrm{F}$ & & MP & & MP & & & & MP & & & \\
\hline
\end{tabular}


Table 2: (continued)

\begin{tabular}{|c|c|c|c|c|c|c|c|c|c|c|c|c|}
\hline \multirow{2}{*}{ Pollen type } & \multirow{2}{*}{ FO } & \multicolumn{11}{|c|}{ Honey samples } \\
\hline & & I & II & III & IV & $\mathbf{V}$ & VI & VII & VIII & IX & $\mathbf{X}$ & XI \\
\hline Anacardium & $\mathrm{R}$ & & & & & & & & & IMP & & \\
\hline Astronium I & $\mathrm{R}$ & & & & & & & & MP & & & \\
\hline Astronium II & $\mathrm{R}$ & & & MP & & & & & & & & \\
\hline Schinus & $\mathrm{F}$ & & & & & & & & & MP & & MP \\
\hline Spondias & $\mathrm{R}$ & & & & & & MP & & & & & \\
\hline S. tuberosa & $\mathrm{F}$ & & MP & & & & & & & MP & MP & \\
\hline S. venulosa & $\mathrm{R}$ & MP & & & & & & & & & & \\
\hline Tapirira guianensis & $\mathrm{R}$ & & & & MP & & & & & & & \\
\hline Aquifoliaceae & & & & & & & & & & & & \\
\hline Ilex & $\mathrm{R}$ & & & MP & & & & & & & & \\
\hline Araliaceae & & & & & & & & & & & & \\
\hline $\begin{array}{l}\text { Didymopanax } \\
\text { macrocarpus } \\
\text { Arecaceae }\end{array}$ & $\mathrm{R}$ & MP & & & & & & & & & & \\
\hline Arecaceae I & $\mathrm{F}$ & & & & & & & MP & & MP & & \\
\hline Cocos & $\mathrm{R}$ & & MP & & & & & & & & & \\
\hline Desmoncus & $\mathrm{R}$ & & MP & & & & & & & & & \\
\hline $\begin{array}{l}\text { Syagrus coronata } \\
\text { Asteraceae }\end{array}$ & VF & MP & MP & & MP & & & MP & & IMP & IMP & IMP \\
\hline Asteraceae I & $\mathrm{R}$ & & MP & & & & & & & & & \\
\hline Asteraceae II & $\mathrm{R}$ & & & & & & & MP & & & & \\
\hline Acanthospermum & $\mathrm{F}$ & & & MP & & & MP & & & & & \\
\hline Austroeupatorium I & $\mathrm{F}$ & & MP & MP & & IMP & MP & & & & & \\
\hline $\begin{array}{l}\text { Austroeupatorium } \\
\text { II }\end{array}$ & $\mathrm{F}$ & & & & & MP & & MP & MP & & & \\
\hline Baccharis & $\mathrm{R}$ & & & & & MP & & & & & & \\
\hline $\begin{array}{l}\text { Centratherum } \\
\text { punctatum }\end{array}$ & $\mathrm{R}$ & & & & & & & & & & & MP \\
\hline Chaptalia & $\mathrm{R}$ & & & MP & & & & & & & & \\
\hline Lepidaploa & VF & MP & MP & MP & MP & MP & & MP & IMP & IMP & & \\
\hline Mikania & $\mathrm{F}$ & & & & & & & & MP & MP & & \\
\hline Moquiniastrum & $\mathrm{F}$ & MP & IMP & MP & & & & & & & & \\
\hline Pluchea sagittalis & VF & IMP & IMP & & MP & MP & & & & & & IMP \\
\hline Senecio I & $\mathrm{R}$ & & & MP & & & & & & & & \\
\hline Senecio II & $\mathrm{R}$ & & & & & & & & & MP & & \\
\hline Tridax procumbens & $\mathrm{F}$ & & & & & & & & & MP & & IMP \\
\hline Vernonanthura I & $\mathrm{F}$ & & MP & IMP & IMP & & & & & & & \\
\hline Vernonanthura II & $\mathrm{F}$ & & MP & & MP & & & & & & & \\
\hline Vernonanthura II & $\mathrm{F}$ & & MP & & MP & & & & & & & \\
\hline $\begin{array}{l}\text { Vernonia } \\
\text { Begoniaceae }\end{array}$ & VF & IMP & IMP & MP & & & & $\mathrm{SP}$ & IMP & IMP & MP & \\
\hline $\begin{array}{l}\text { Begonia } \\
\text { Bignoniaceae }\end{array}$ & $\mathrm{R}$ & & & & & & & MP & & & & \\
\hline Bignoniaceae I & $\mathrm{R}$ & & & & MP & & & & & & & \\
\hline Jacaranda & $\mathrm{R}$ & & & & & & & & & IMP & & \\
\hline $\begin{array}{l}\text { Xylophragma } \\
\text { myrianthum } \\
\text { Brassicaceae }\end{array}$ & $\mathrm{F}$ & & & & MP & & MP & & & & & \\
\hline Brassica & $\mathrm{R}$ & MP & & & & & & & & & & \\
\hline
\end{tabular}


Table 2: (continued)

\begin{tabular}{|c|c|c|c|c|c|c|c|c|c|c|c|c|}
\hline \multirow{2}{*}{ Pollen type } & \multirow{2}{*}{ FO } & \multicolumn{11}{|c|}{ Honey samples } \\
\hline & & I & II & III & IV & $\mathbf{V}$ & VI & VII & VIII & IX & $\mathbf{X}$ & XI \\
\hline Cactaceae & & & & & & & & & & & & \\
\hline $\begin{array}{l}\text { Melocactus } \\
\text { zehntneri } \\
\text { Combretaceae }\end{array}$ & $\mathrm{R}$ & & MP & & & & & & & & & \\
\hline Combretum & $\mathrm{R}$ & & & & & & & & & & MP & \\
\hline Convolvulaceae & & & & & & & & & & & & \\
\hline Evolvulus & $\mathrm{R}$ & & & & & MP & & & & & & \\
\hline E. elaeagnifolius & $\mathrm{R}$ & & MP & & & & & & & & & \\
\hline E. nuтmularius & $\mathrm{R}$ & MP & & & & & & & & & & \\
\hline Odonellia & $\mathrm{R}$ & & & MP & & & & & & & & \\
\hline Cucurbitaceae & & & & & & & & & & & & \\
\hline $\begin{array}{l}\text { Momordica } \\
\text { charantia } \\
\text { Euphorbiaceae }\end{array}$ & $\mathrm{R}$ & & & & & & & & & & & IMP \\
\hline Euphorbiaceae I & $\mathrm{F}$ & & & & MP & & MP & & & & & \\
\hline Cnidoscolus & $\mathrm{R}$ & & & & & & & & & & & MP \\
\hline C. urens & $\mathrm{F}$ & & & & & & MP & & & & IMP & \\
\hline Croton I & $\mathrm{R}$ & & MP & & & & & & & & & \\
\hline Croton II & $\mathrm{R}$ & & & & & & & & & MP & & \\
\hline C. echioides & $\mathrm{F}$ & MP & & MP & & & & & & & & \\
\hline C. lundianus & $\mathrm{R}$ & & & & MP & & & & & & & \\
\hline Manihot & $\mathrm{R}$ & & & & MP & & & & & & & \\
\hline M. tripartita & $\mathrm{R}$ & MP & & & & & & & & & & \\
\hline Pausandra & $\mathrm{F}$ & & IMP & & & & & & & & & MP \\
\hline $\begin{array}{l}\text { Sapium } \\
\text { glandulosum } \\
\text { Fabaceae }\end{array}$ & $\mathrm{R}$ & & & & & & & MP & & & & \\
\hline Aeschynomene & $\mathrm{R}$ & & IMP & & & & & & & & & \\
\hline $\begin{array}{l}\text { Anadenanthera } \\
\text { colubrina }\end{array}$ & $\mathrm{R}$ & & & & & & MP & & & & & \\
\hline Andira & $\mathrm{R}$ & MP & & & & & & & & & & \\
\hline Caesalpinia & $\mathrm{R}$ & & & & & & & & MP & & & \\
\hline Cajanus cajan & $\mathrm{R}$ & & MP & & & & & & & & & \\
\hline $\begin{array}{l}\text { Cenostigma } \\
\text { pyramidale }\end{array}$ & $\mathrm{R}$ & & & & & & MP & & & & & \\
\hline Chamaecrista & $\mathrm{R}$ & & & & & & & & MP & & & \\
\hline C. nictitans & $\mathrm{R}$ & & & & & & & & IMP & & & \\
\hline C. ramosa & $\mathrm{F}$ & & MP & & & & & & IMP & & & \\
\hline C. swainsonii & $\mathrm{F}$ & & MP & MP & & & & & & & & \\
\hline Dalbergia & $\mathrm{R}$ & & & & & & & & & MP & & \\
\hline $\begin{array}{l}\text { Indigofera } \\
\text { microcarpa }\end{array}$ & $\mathrm{R}$ & & & & & & & & MP & & & \\
\hline Leucaena & $\mathrm{R}$ & & & & & MP & & & & & & \\
\hline Mimosa & $\mathrm{F}$ & & & IMP & & MP & & & & & & \\
\hline M. arenosa & $\mathrm{VF}$ & $\mathrm{SP}$ & SP & $\mathrm{PP}$ & IMP & IMP & & MP & & MP & IMP & \\
\hline M. caesalpiniifolia & VF & IMP & & IMP & & & MP & & SP & & & MP \\
\hline M. candolei & VF & MP & MP & MP & & & & MP & IMP & MP & & \\
\hline M. sensitiva & VF & SP & MP & IMP & PP & PP & & IMP & MP & IMP & & IMP \\
\hline M. setosa & $\mathrm{R}$ & & & & & & & MP & & & & \\
\hline M. setuligera & $\mathrm{R}$ & & IMP & & & & & & & & & \\
\hline
\end{tabular}


Table 2: (continued)

\begin{tabular}{|c|c|c|c|c|c|c|c|c|c|c|c|c|}
\hline \multirow{2}{*}{ Pollen type } & \multirow{2}{*}{ FO } & \multicolumn{11}{|c|}{ Honey samples } \\
\hline & & I & II & III & IV & $\mathbf{V}$ & VI & VII & VIII & IX & $\mathbf{X}$ & $\mathbf{X I}$ \\
\hline M. tenuiflora & $\mathrm{VF}$ & MP & MP & & MP & MP & & IMP & MP & IMP & IMP & IMP \\
\hline M. verrucosa & $\mathrm{R}$ & & & & & & & & MP & & & \\
\hline Parapiptadenia & $\mathrm{R}$ & & & & & & & MP & & & & \\
\hline Piptadenia & $\mathrm{F}$ & & & & & & & & & MP & MP & \\
\hline $\begin{array}{l}\text { Plathymenia } \\
\text { reticulata }\end{array}$ & $\mathrm{R}$ & & & & MP & & & & & & & \\
\hline Poeppigia procera & $\mathrm{R}$ & MP & & & & & & & & & & \\
\hline Prosopis & $\mathrm{VF}$ & & & & MP & & & MP & MP & MP & MP & \\
\hline Pterocarpus rohrii & $\mathrm{F}$ & & & & & & & MP & & & & IMP \\
\hline Senegalia bahiensis & $\mathrm{VF}$ & MP & MP & & & MP & & & & MP & MP & MP \\
\hline S. tenuifolia & $\mathrm{R}$ & & & & MP & & & & & & & \\
\hline Senna & $\mathrm{F}$ & & & & & & & & & IMP & IMP & \\
\hline S. macranthera & $\mathrm{R}$ & & & & & & MP & & & & & \\
\hline $\begin{array}{l}\text { S. spectabilis } \\
\text { Lamiaceae }\end{array}$ & $\mathrm{F}$ & & & & & & $\mathrm{PP}$ & IMP & & & & \\
\hline $\begin{array}{l}\text { Eplingiella } \\
\text { fruticosa }\end{array}$ & $\mathrm{F}$ & & MP & & MP & & & & & & MP & \\
\hline Eriope blanchetii & $\mathrm{R}$ & & & & & & MP & & & & & \\
\hline $\begin{array}{l}\text { Salvia } \\
\text { Malvaceae }\end{array}$ & $\mathrm{F}$ & & & & & & & MP & & IMP & IMP & MP \\
\hline Bombacoideae & $\mathrm{R}$ & & & & & & IMP & & & & & \\
\hline Ceiba & $\mathrm{R}$ & & & & & & MP & & & & & \\
\hline Herissantia & $\mathrm{VF}$ & MP & MP & MP & MP & MP & IMP & MP & IMP & MP & MP & MP \\
\hline $\begin{array}{l}\text { Melochia } \\
\text { tomentosa }\end{array}$ & $\mathrm{F}$ & MP & & & & & & & MP & & & \\
\hline Sida cordifolia & $\mathrm{R}$ & & & & & & & & MP & & & \\
\hline S. galheirensis & $\mathrm{R}$ & & & & & & & & & & MP & \\
\hline $\begin{array}{l}\text { Sidastrum } \\
\text { micranthum }\end{array}$ & $\mathrm{F}$ & MP & MP & & MP & & & & & MP & & \\
\hline S. paniculatum & $\mathrm{R}$ & & & & & MP & & & & & & \\
\hline $\begin{array}{l}\text { Waltheria } \\
\text { Melastomataceae }\end{array}$ & $\mathrm{F}$ & & & & & & & & MP & & IMP & \\
\hline Clidemia & $\mathrm{R}$ & & & & & & & & & & MP & \\
\hline C. hirta & $\mathrm{F}$ & & & & & MP & & & MP & & & \\
\hline Miconia & $\mathrm{R}$ & & & & & MP & & & & & & \\
\hline Microteaceae & & & & & & & & & & & & \\
\hline $\begin{array}{l}\text { Microtea } \\
\text { Molluginaceae }\end{array}$ & $\mathrm{F}$ & & & & & & & MP & & & MP & \\
\hline $\begin{array}{l}\text { Mollugo verticillata } \\
\text { Myrtaceae }\end{array}$ & $\mathrm{R}$ & & & & & & & & MP & & & \\
\hline Campomanesia & $\mathrm{R}$ & & MP & & & & & & & & & \\
\hline Eucalyptus & VF & MP & MP & MP & MP & MP & MP & & & MP & MP & \\
\hline Eugenia & $\mathrm{R}$ & & & & & & & & & & IMP & \\
\hline Myrcia & $\mathrm{F}$ & MP & & & & & & & & & MP & IMP \\
\hline M. selloi & $\mathrm{F}$ & MP & & & & & & & & & & MP \\
\hline Myrciaria & $\mathrm{R}$ & & & & MP & & & & & & & \\
\hline $\begin{array}{l}\text { Psidium guajava } \\
\text { Nyctaginaceae }\end{array}$ & $\mathrm{F}$ & & & MP & MP & & & & & & & \\
\hline Boerhavia & $\mathrm{R}$ & & & & & & MP & & & & & \\
\hline $\begin{array}{l}\text { B. diffusa } \\
\text { Onagraceae }\end{array}$ & $\mathrm{R}$ & & & & & & & & MP & & & \\
\hline Ludwigia & $\mathrm{R}$ & & & & MP & & & & & & & \\
\hline
\end{tabular}


Table 2: (continued)

\begin{tabular}{|c|c|c|c|c|c|c|c|c|c|c|c|c|}
\hline \multirow{2}{*}{ Pollen type } & \multirow{2}{*}{ FO } & \multicolumn{11}{|c|}{ Honey samples } \\
\hline & & $\mathbf{I}$ & II & III & IV & $\mathbf{V}$ & VI & VII & VIII & IX & $\mathbf{X}$ & XI \\
\hline \multicolumn{13}{|l|}{ Plantaginaceae } \\
\hline $\begin{array}{l}\text { Mecardonia } \\
\text { Poaceae }\end{array}$ & $\mathrm{F}$ & & & & & & & & & IMP & IMP & MP \\
\hline Poaceae I & $\mathrm{F}$ & MP & & & MP & & & & & & & \\
\hline Poaceae II & $\mathrm{R}$ & & & MP & & & & & & & & \\
\hline Andropogon & $\mathrm{R}$ & & & & & & & & & MP & & \\
\hline Digitaria & $\mathrm{F}$ & & & MP & & & MP & & & & & \\
\hline Trachypogon & VF & MP & IMP & & MP & & & & & & MP & IMP \\
\hline \multicolumn{13}{|l|}{ Polygonaceae } \\
\hline Polygonит & $\mathrm{R}$ & & & MP & & & & & & & & \\
\hline \multicolumn{13}{|l|}{ Rhamnaceae } \\
\hline $\begin{array}{l}\text { Sarcomphalus } \\
\text { joazeiro }\end{array}$ & $\mathrm{R}$ & & & & $\mathrm{MP}$ & & & & & & & \\
\hline \multicolumn{13}{|l|}{ Rubiaceae } \\
\hline Borreria & $\mathrm{R}$ & & & & MP & & & & & & & \\
\hline B. verticillata & VF & MP & MP & MP & & MP & MP & $\mathrm{PP}$ & IMP & IMP & IMP & \\
\hline Diodia & $\mathrm{R}$ & & & & & & MP & & & & & \\
\hline Hexasepalum teres & $\mathrm{F}$ & & & & & & & & IMP & & MP & \\
\hline Mitracarpus & $\mathrm{F}$ & & & MP & MP & & & & & IMP & IMP & \\
\hline M. baturitensis & $\mathrm{F}$ & & & & & & & MP & & IMP & & \\
\hline M. strigosus & $\mathrm{F}$ & MP & & & & MP & & & & & & \\
\hline $\begin{array}{l}\text { Richardia } \\
\text { grandiflora } \\
\text { Salicaceae }\end{array}$ & $\mathrm{F}$ & & & & & & & & & MP & IMP & \\
\hline Casearia sylvestris & $\mathrm{R}$ & & & & MP & & & & & & & \\
\hline Sapindaceae & & & & & & & & & & & & \\
\hline Cardiospermum & $\mathrm{R}$ & MP & & & & & & & & & & \\
\hline $\begin{array}{l}\text { Serjania lethalis } \\
\text { Solanaceae }\end{array}$ & $\mathrm{F}$ & & & MP & & & & & & $\mathrm{MP}$ & & \\
\hline Solanum & $\mathrm{R}$ & MP & & & & & & & & & & \\
\hline $\begin{array}{l}\text { S. paniculatum } \\
\text { Urticaceae }\end{array}$ & $\mathrm{F}$ & & & & & & & MP & & & MP & IMP \\
\hline Boehmeria & $\mathrm{R}$ & MP & & & & & & & & & & \\
\hline $\begin{array}{l}\text { Cecropia } \\
\text { pachystachya } \\
\text { Zygophyllaceae }\end{array}$ & $\mathrm{F}$ & MP & & & MP & & & & & & & IMP \\
\hline Kallstroemia & $\mathrm{R}$ & & & & & & MP & & & & & \\
\hline
\end{tabular}

Only one pollen type appeared in $100 \%$ of the samples: Herissantia type. The remaining most frequent pollen types (>45\%) were Borreria verticillata (Rubiaceae), Mimosa sensitiva and $M$. tenuiflora (Fabaceae), in nine samples; Eucalyptus (Myrtaceae), Lepidaploa (Asteraceae) and $M$. arenosa (Fabaceae), in eight samples; Alternanthera brasiliana (Amaranthaceae), Syagrus coronata (Arecaceae) and Vernonia (Asteraceae), in seven samples; M. candollei and Senegalia bahiensis (Fabaceae), in six samples; and M. caesalpiniifolia (Fabaceae), Pluchea sagittalis (Asteraceae), Prosopis (Fabaceae) and Trachypogon (Poaceae) in five samples (Figure 4).

Herissantia type was found as important minor pollen (IMP) in the samples VI and VIII, and minor pollen (MP) in the other samples. The M. tenuiflora type was present in all analyzed samples from the city of Antônio Gonçalves and is classified as IMP and MP, while the Eucalyptus type occurred in all samples from Campo Formoso as IMP. 
Of the 11 samples analyzed, five had the predominant pollen type $(>45 \%)$ and four had secondary pollen (16-45\%). The pollen types B. verticillata and Vernonia occurred in sample VII as predominant (PP) and secondary (SP), respectively; Mimosa arenosa and M. sensitiva were found in sample I as SP and, in other samples (samples III/M. arenosa, IV and V/M. sensitiva), as PP; M. caesalpiniifolia was classified as SP in sample VIII and Senna spectabilis as PP in sample VI.

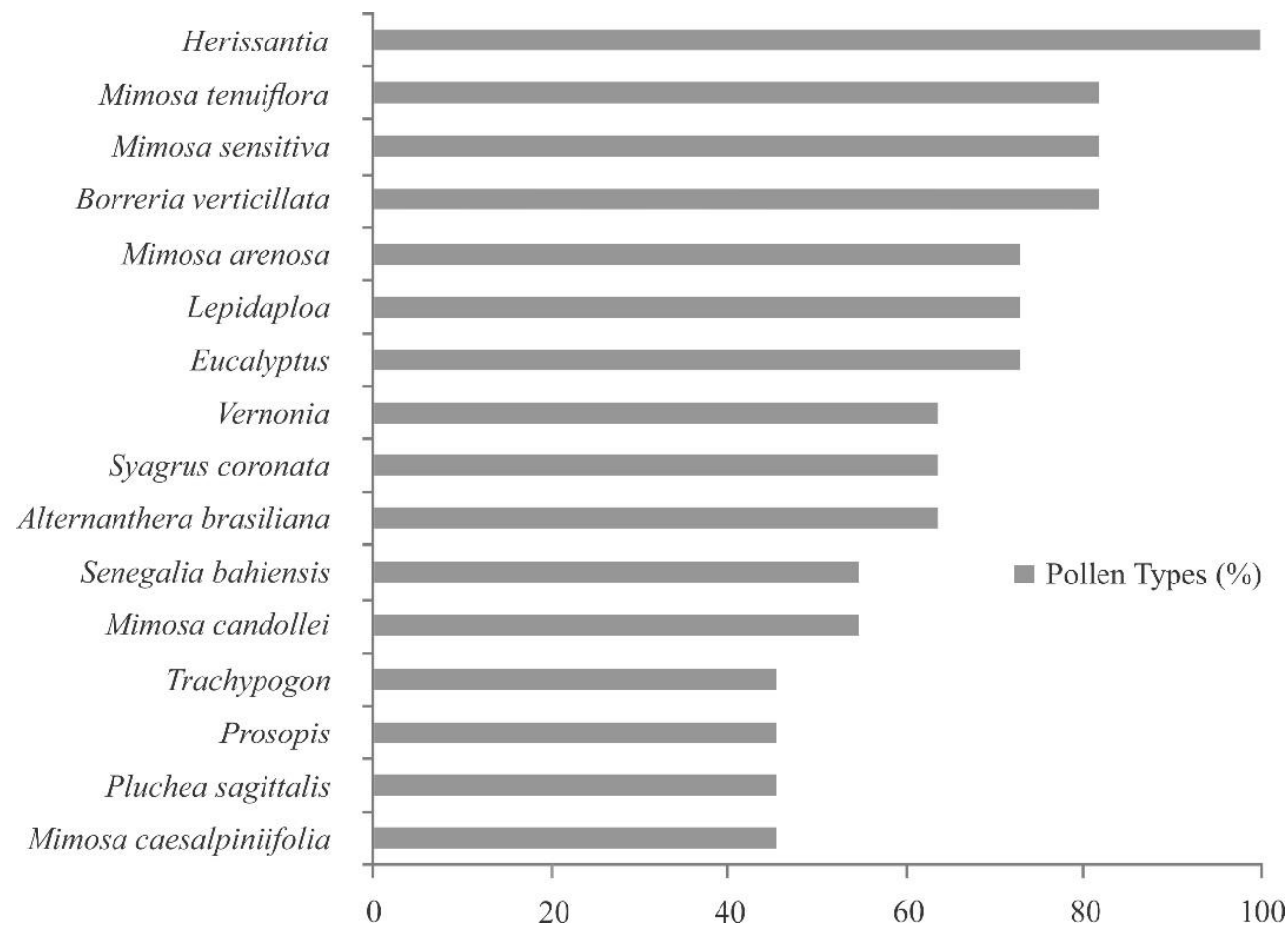

Figure 4: Pollen types present in more than $45 \%$ of the total samples analyzed $(n=11)$.

Among the pollen types occurring as IMP, the most common in the studied samples were: $M$. sensitiva, M. tenuiflora, and Vernonia in four samples; B. verticillata, M. arenosa, Pluchea sagittalis, and Syagrus coranota in three samples. In addition, B. verticillata and M. tenuiflora were found in five samples as MP, the majority (85.72\%) from the city of Campo Formoso.

Regarding the richness of pollen type in the samples, the greatest number was found in samples II (38 types) and I (36 types) from the city of Campo Formoso. The $M$. arenosa type characterized the pollen spectrum of these samples, occurring in both as SP $(24.8 \%$ in sample I and $29.9 \%$ in sample II). The lowest frequency of types was found in samples V (20 types) and XI (21 types), produced in September (2014) and May (2016), respectively. The average value of pollen types per sample was 28.18 .

The total pollen diversity was 3443 nats.ind- 1 and 0.9274 for dominance. Considering the Shannon-Weiner index, sample IX was the more diverse, whereas samples IV, V and VI were the less diverse. For Simpson index, samples IX, X and XI presented higher diversity, while IV, V and VI showed lower diversity (Table 3). It is worth note that diversity is not related to the geographical distance. 
Table 3: Diversity indices from honey samples: Values followed by the same letters, in the same column, did not differ among themselves at the level of $5 \%$ of significance.

\begin{tabular}{ccc}
\hline Samples & Shannon-Weiner & Simpson \\
\hline I & $2.338 \mathrm{a}$ & $0.8153 \mathrm{a}$ \\
II & $2.66 \mathrm{a}$ & $0.8727 \mathrm{a}$ \\
III & $1.507 \mathrm{~b}$ & $0.5943 \mathrm{~b}$ \\
IV & $1.26 \mathrm{c}$ & $0.4308 \mathrm{c}$ \\
V & $1.043 \mathrm{c}$ & $0.4059 \mathrm{c}$ \\
VI & $1.162 \mathrm{c}$ & $0.4392 \mathrm{c}$ \\
VII & $1.938 \mathrm{~b}$ & $0.7433 \mathrm{~b}$ \\
VIII & $2.679 \mathrm{a}$ & $0.8988 \mathrm{a}$ \\
IX & $3.172 \mathrm{~d}$ & $0.9491 \mathrm{~d}$ \\
X & $2.865 \mathrm{a}$ & $0.9276 \mathrm{~d}$ \\
XI & $2.613 \mathrm{a}$ & $0.9154 \mathrm{~d}$ \\
\hline Total & 3.443 & 0.9274 \\
\hline
\end{tabular}

In the similarity analysis, the samples from the Antônio Gonçalves city showed a lower index when compared to each other. Samples IX (December 2015) and X (May 2016) were the most similar concerning the pollen types found, with approximately $38 \%$ similarity and 17 pollen types in common (Figure 5). Sample VII (October 2014) showed a similarity of $33 \%$ and $26 \%$ with samples IX and X, respectively. It is worth to note that the samples were divided in two different groups. The first one comprises all samples from Campo Formoso (I, II, III, IV, V, VI) and one from Antônio Gonçalves (VII), whereas the second groups is formed by four samples from Antônio Gonçalves (VIII, IX, X e XI), which indicates that samples geographically closest to each other share many pollen types.

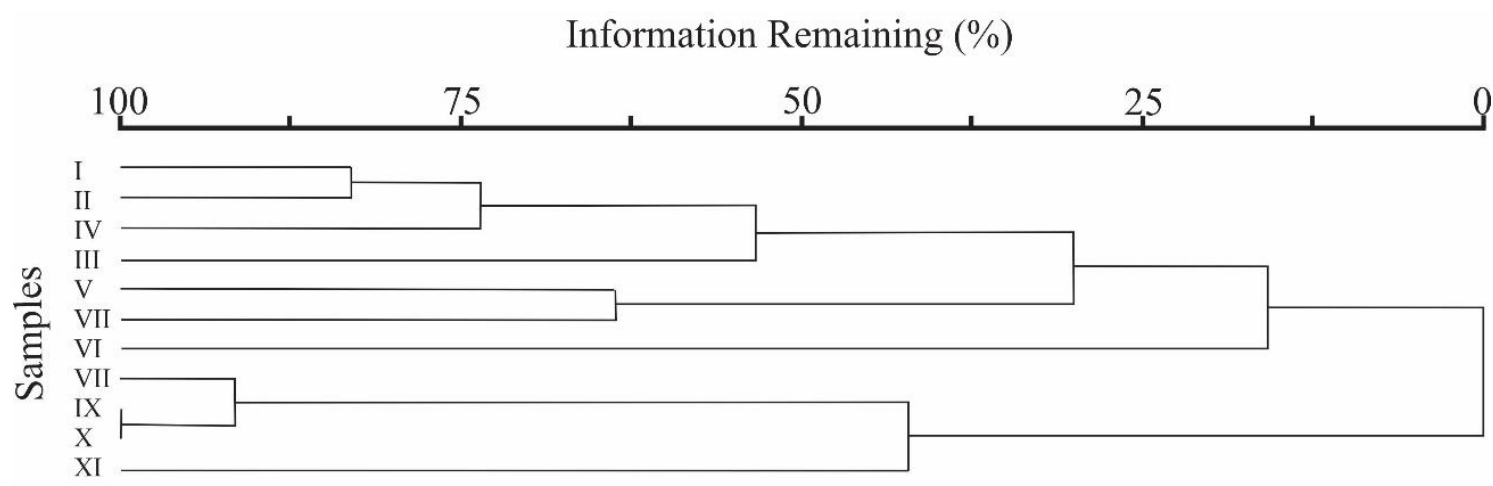

Figure 5: Similarity dendrogram (Jaccard index) between samples (I - XI) of honeys produced in the cities of Antônio Gonçalves and Campo Formoso, Bahia, Brazil.

However, considering the species in common, the greatest similarity (20\%) was found between samples V (Campo Formoso) and VII (Antônio Gonçalves), and the shared pollen types were Alternanthera brasiliana, Austroeupatorium II, Borreria verticillata, Herissantia, Lepidaploa, Mimosa arenosa, M. pudica and M. tenuiflora, which could be related with the temporal approach, since they were collected in the same year (2014).

\section{DISCUSSION}

The presence of pollen types from different botanical sources confirms an easy adaptation and the forage exploitation behavior of A. mellifera in different environments [41]. Aguiar et al. (2003) 
[42] argued that, for nectar sources, it seems to predominate a pattern of visits to various plants, which corroborates the results presented here.

What normally occurs in the Northeast of Brazil is that the honey takes a "wild characteristic" due to the great variation of nectar sources [43]. The availability of this floral resource, together with the generalist behavior of bees, makes plant species are frequently foraged according to their flowering period at different times of the year. Demartelaere et al. (2010) [44] stated that the first step towards a better rationalization of beekeeping is to know the plant species used by bees as such as source of nectar and pollen.

A great diversity of pollen types was found in the pollen spectrum of the honeys of Antônio Gonçalves and Campo Formoso, Bahia. The majority of these pollen types is from typical species of the Caatinga flora, all species are from variated botanical families, but some are endemic and others are broadly occurring, such as Spondias tuberosa Arruda (Anacardiaceae) and Sarcomphalus joazeiro (Mart.) Hauenshild (Rhamnaceae) (endemic), and with the predominance of the Fabaceae, Anadenanthera colubrina (Vell.) Brenan, Chamaecrista swainsonii (Benth.) H.S.Irwin \& Barneby (endemic), Senegalia bahiensis (Benth.) Seigler \& Ebinger (endemic), and Mimosa caesalpiniifolia Benth. $[45,46]$.

In Brazil, the family Fabaceae is represented by approximately 222 genera and 2,848 species [47]. The wide diversity of this family confirms its important contribution to the survival and maintenance of bees of the species A. mellifera, both in the number of pollen types as recorded here and in the pollen frequency of samples. This importance has also been mentioned by other authors [27, 48]. Sodré (2005) [49], in characterizing the honeys of A. mellifera from the states of Ceará and Piauí, also found Fabaceae as the most representative family in the number of species.

Oliveira et al. (2010) [22] analyzed the A. mellifera honey produced in a Caatinga area of the Nova Soure city, Bahia, and recorded the families Mimosaceae (Fabaceae, 11 types), Caesalpiniaceae (Fabaceae, nine types), Fabaceae (five types), and Rubiaceae (five types) as the most representative in the number of pollen types. In their research on the botanical origin of pollen loads collected by A. mellifera in the city of Canavieiras, Bahia, Dórea et al. (2010) [21] stressed that the Fabaceae (with nine types) presented the greatest richness of pollen types, followed by Asteraceae (four types), Euphorbiaceae (four types), and Anacardiaceae (two types).

The results presented here concerning the main botanical families used as resources by Africanized bees corroborate the data found in Oliveira et al. (2010) [22], in which Mimosaceae and Lamiaceae were present in all samples analyzed, followed by Asteraceae, Rubiaceae, and Caesalpiniaceae (94\% each), Amaranthaceae (88.2\%), Fabaceae (76.5\%), and Anacardiaceae, Myrtaceae and Sapindaceae (58.8\% each). Additionally, Santos et al. (2006) [50] included the occurrence of Fabaceae (75), Asteraceae (46), Euphorbiaceae (43), Malvaceae (43) and Rubiaceae (26) in the list of species that comprise bee pasture in the semiarid region.

In other surveys carried out in Bahia, Nascimento et al. (2014) [51] also recorded that Fabaceae presented the highest species richness (22\%), followed by Asteraceae (14\%) and Malvaceae (7\%), of the total number of species visited by A. mellifera in the Recôncavo Baiano region. Vidal et al. (2008) [52] recorded Compositae (Asteraceae, eight species), Verbenaceae (five species), Leguminosae (four species), Myrtaceae (three species), and Rubiaceae (three species) for the bee flora of five cities in Bahia (Cruz das Almas, Governador Mangabeira, Muritiba, São Félix, and Cachoeira).

During their study on the collection of trophic resources by bees, Ramalho et al. (1990) [53] stated that Asteraceae, Arecaceae, Euphorbiaceae, Rubiaceae and Sterculiaceae (Malvaceae) are among the main botanical families for A. mellifera in the Neotropical region. Some other studies related to the bee flora indicate that species of Asteraceae, Fabaceae, Malvaceae, Rubiaceae and Solanaceae are important resources for bees [54, 55].

The contribution of the genus Mimosa (Fabaceae) to the composition of the honey in the two cities studied was quite significant. Several studies dealing with the botanical origin of honeys and carried out in the State of Bahia recognize the importance of this genus for the production [11, 17, 21, 22, 24, 56]. According to Oliveira and Santos (2014) [11], the participation of pollen types of the genus Mimosa in honey samples from Bahia may be related to their distribution and diversity in the state and, consequently, its greater availability for bees. 
The studies carried out by Buril et al. (2010) [34] on the pollen diversity of the Mimosoideae (Fabaceae) in an area of the Caatinga and by Lima et al. (2006) [28] on the pollen morphology of Mimosa species accessed by bees in the semiarid region contributed to the recognition of differences in the pollen morphology among species. As a consequence, considering the morphological similarities of the pollen grains of Mimosa species in the analysis, it was possible to group them into different pollen types and subtypes.

Thus, in the occurrence of the Mimosa sensitiva pollen type (one of the most frequent among the samples analyzed here), the pollen of M. hypoglauca Mart., M. pudica L., M. sensitiva L. var. sensitiva, and M. velloziana Mart. may also have occurred due to the botanical affinity of these species [28]. Likewise, the types M. tenuiflora and M. caesalpiniifolia considered here may also represent, respectively, the species M. invisa Mart. ex Colla. and M. arenosa (Willd.) Poir. var. arenosa $[28,33,34]$, with the possibility of increasing the number of species (pollen types) registered for the genus Mimosa.

Sodré et al. (2008) [20] classified the Mimosa caesalpiniifolia type as dominant pollen (DP $>45 \%$ ) and accessory pollen (AP - 16-45\%) among the honey samples of A. mellifera in Piauí. In the North Coast, State of Rio Grande do Sul, Nobre et al. (2015) [4] found a predominance of the type Mimosa sp. (41.2\%) in the honey of a polyfloral origin. Sodre et al. (2007) [19] identified the types of M. caesalpiniifolia as dominant pollen and M. verrucosa as occasional isolated pollen (OIP $-<3 \%$ ) in honey samples produced in two states in the Northeast region. Santos et al. (2020) [26] analyzing the honey of bees from the semiarid region in the State of Bahia, found that the type $M$. caesalpiniifolia was frequent in the honey of Melipona quadrifasciata anthidioides and Apis mellifera. The M. arenosa, M. candollei (M. quadrivalvis), and M. setosa were the DP, OIP and AP types, respectively, in stingless bee honey samples from the Bahian semiarid [16].

The Asteraceae family also featured in the pollen spectrum of honeys analyzed in this work. Luz et al. (2007) [57] studied the trophic resources of A. mellifera in the region of Morro Azul do Tinguá, Rio de Janeiro, and found several Asteraceae genera in the samples contributing as DP and with lower percentages. The representativeness of Asteraceae probably results from the fact that the family is one of the largest and most widely distributed among Angiosperms. Also, it is an important source of nectar and pollen $[58,59]$, which probably makes it very accessible by bees.

For Barth (1989) [12], concerning occurrence percentages and classification of the frequency of pollen types among the samples analyzed, the Brazilian honey is characterized by a large amount of pollen in the isolated category, as recorded here. Although these parameters have little importance in the amount of nectar supplied, they are of interest in the geographic determination of honey. According to Borges et al. (2006) [27], the frequency of a pollen type may vary seasonally throughout the year and with the geographic area in focus.

Oliveira and Santos (2014) [11] analyzed the honey of A. mellifera in the State of Bahia and found that the types Alternanthera (53.7\%), Borreria verticillata (64.2\%), Mimosa arenosa (65.7\%), M. sensitiva (83.6\%), and M. tenuiflora (68.7\%) occurred in most samples and classified them in the very frequent category.

Moreti et al. (2005) [18] found among the 41 pollen types found in samples of honey of $A$. mellifera collected in the State of Ceará the Borreria verticillata type as DP or AP, and the Eucalyptus type as OIP and important isolated pollen (IIP - 3 a 15\%). Aires and Freitas (2001) [60] found the types of Borreria verticillata, Mimosa caesalpiniifolia, and M. tenuiflora as the most frequent in the analyzed samples. These data corroborate those recorded in the present study.

Borreria verticillata (L.) G.Mey., Mimosa arenosa (Willd.) Poir. and M. tenuiflora (Willd.) Poir. are considered by several authors as excellent suppliers of nectar and pollen for bees $[1,11,18,50$, 61-63]. This reinforces the great apicultural potential of these species and their representativeness for the studied region; however, according to Barth (1989) [12], B. verticillata and species of the genus Mimosa are considered over-represented plants in the pollen spectra, providing little nectar but a lot of pollen.

In this regard, as the pollen grains of $M$. arenosa, $M$. sensitiva and $M$. tenuiflora are considered small [33], they can be over-represented in the samples due to the criterion adopted for counting, which took into account only the number of pollen grains in the sample, disregarding the volume occupied by each of them. Andrade et al. (2009) [64] also pointed out that dominant types identified 
as pollinic sources can be over-considered, while under-considered pollen types may be more important in identifying the floral origin of a honey sample.

Similar results for diversity of honey samples were found by Sekine et al. (2013) [65] in Paraná State, Brazil and Santos et al. (2020) [26] in Bahia State, Brazil. The high values verified by Simpson Index indicate the probability of diversity of the sampled pollen due to the dominance of one or a few species, for instance, $M$. sensitiva, M. tenuiflora, Senna sp. and B. verticillata. Pollen diversity and geographical distance were probably the main factors to separate samples in the similarity analysis, although they were collected in different time of the year. Adequate knowledge on pollen diversity from melissopalynological analyses is indispensable for qualifying the honey samples.

Based on the similarity analysis of the pollen spectrum of samples from the study area it can be inferred that the bees A. mellifera share several plant species as a resource for honey production. However, some differences observed in this index may be related to the number of pollen types classified as MP, which represent $74.20 \%$ of the total pollen types distributed in the different frequency classes among samples. As stated by Moreti et al. (2000) [17], this similarity analysis considers the distribution of the flora throughout the year, as well as the number of samples analyzed, which does not allow a standardized pollen characterization among the samples of the cities.

\section{CONCLUSION}

The variety of pollen types found in honeys from the cities of Antônio Gonçalves and Campo Formoso, Bahia, revealed important characteristics of the flora and a high diversity of species and botanical families with melliferous potential in the region, allowing its classification as multifloral honeys. Such characteristics justify the flexibility of foraging exploitation of Apis mellifera to different families and botanical species, in addition to its ability to adapt to different environments.

Knowing plant species of bee value in the region is essential for the establishment of qualified, productive and sustainable beekeeping. Therefore, it is necessary to carry out studies on the local and regional bee flora to determine its foraged resources, as well as its pollen grains morphology. Such information may support future related works, for example, in the Melissopalynology.

Regarding the presence of pollen types in the analyzed samples, Fabaceae and Asteraceae, followed by Euphorbiaceae, Anacardiaceae, Malvaceae and Rubiaceae, contributed significantly to the composition of the honeys, becoming the main resources accessed by A. mellifera in the cities. The pollen types Borreria verticillata, Mimosa sensitiva, and M. tenuiflora were present in $81.82 \%$ of the samples, while Eucalyptus, Lepidaploa, and M. arenosa were found in $72.73 \%$.

The genus Mimosa, with eight recorded pollen types and an expressive frequency in $100 \%$ of the samples, was representative in the pollen spectrum of the honeys. These findings indicate the importance of the genus as a bee resource in the studied region.

The present study contributed to the recognition of the most exploited nectariferous and/or polliniferous species of plants by Apis mellifera in the region. Therefore, it becomes an important tool to assist in the preservation and propagation of these plants and, consequently, increasing honey production. Finally, the final value of the product on the market can also benefit from the correct certification of its botanical origin.

\section{ACKNOWLEDGMENTS}

The authors thank Fundação de Amparo à Pesquisa do Estado da Bahia (FAPESB) for the financial support, the Cooperativa Apícola e Pesqueira de Campo Formoso (COOAPCAF) for supplying honey samples, the Laboratório de Micromorfologia Vegetal (LAMIV) for allowing access to the pollen collection to compare and identify the pollen grains, and the researchers Dr. Paulino Oliveira and Dr. Francisco de Assis Ribeiro dos Santos for their assistance in identifying pollen types. 


\section{BIBLIOGRAPHIC REFERENCES}

1. Freitas BM, Silva EMS. Potencial apícola da vegetação do semi-árido brasileiro. In: Santos FAR, editor. Apium Plantae. Recife (PE): IMSEAR; 2006. p. 19-32.

2. Costa COM, Freitas FRD. A produção de mel de abelha (Apis mellifera) no município de Jardim: um estudo de caso. Cad Cultura Ciên. 2009;1(1):56-76.

3. Santos CS, Ribeiro AS. Apicultura uma alternativa na busca do desenvolvimento sustentável. Rev Verde Agroecol Desenv Sustentável. 2009 Jul/Sep;4(3):1-6.

4. Nobre SB, Bauermann SG, Lopes LA, Evaldt ACP. Características polínicas de méis de Apis mellifera L., 1758 (Hymenoptera, Apidae, Apini) do Litoral Norte, estado do Rio Grande do Sul, Brasil. Rev Ciên Amb. 2015;9(1):87-100.

5. Wolff LF. Sistema de produção de mel para a região sul do Rio Grande do Sul. Pelotas (RS): Embrapa Clima Temperado; 2018. 90 p.

6. Ponciano NJ, Golynski A, Souza PM, Ney MG, Ney VSP. Características do nível tecnológico dos apicultores do estado do Rio de Janeiro. RESR. 2013 Jul/Sept;51(3):499-514, doi: 10.1590/S010320032013000300005.

7. Almeida MAD, Carvalho CMS. Apicultura: uma oportunidade de negócio sustentável. Salvador (BA): Sebrae; 2009. $52 \mathrm{p}$.

8. Reis VDA, Comastri Filho JA. Importância da Apicultura no Pantanal Sul-Mato Grossense. Corumbá (MS): Documentos/Embrapa Pantanal; 2003. 22 p.

9. Silva CI, Aleixo KP, Nunes-Silva B, Freitas BM, Imperatriz-Fonseca VL. Guia ilustrado de abelhas polinizadoras no Brasil. São Paulo: Instituto de Estudos Avançados - Ministério do Meio Ambiente; 2014. $54 \mathrm{p}$.

10. Correia-Oliveira ME, Nunes LA, Silveira TA, Marchini LC, Silva JWP. Manejo da agressividade de abelhas africanizadas. Piracicaba, São Paulo: ESALQ - Divisão de Biblioteca; 2012. 46 p.

11. Oliveira PP, Santos FAR. Prospecção palinológica em méis da Bahia. Feira de Santana (BA): Print Mídia Editora; 2014. 120 p.

12. Barth OM. O pólen no mel brasileiro. Rio de Janeiro: Gráfica Luxor; 1989. 93 p.

13. Barth OM. Análise polínica de mel: avaliação de dados e seu significado. Mensagem Doce (Apacame). 2005 May [cited 2020 Oct 19];81. Available from: http://www.apacame.org.br/mensagemdoce/81/artigo.htm

14. Nordi JC. Flora apícola e polinização. Taubaté (SP): Sistema Integrado de Bibliotecas/UNITAU; 2015. $94 \mathrm{p}$.

15. Vilela SLO. Principais resultados do estudo da cadeia produtiva do mel de abelhas do estado do Piauí: pesquisa socioeconômica. Rev Cient Prod Anim. 2000;2:249-259.

16. Santana ALA, Fonseca AAO, Alves RMO, Carvalho CAL, Melo PA, Silva ES, Souza BA, Jesus JNJ, Sodré GS. Tipos polínicos em amostras de méis de abelhas sem ferrão de municípios do semiárido baiano. Magistra. $2011 \mathrm{Jul} / \mathrm{Sep} ; 23(3): 134-139$.

17. Moreti ACCC, Carvalho CAL, Marchini LC, Oliveira PCF. Espectro polínico de amostras de mel de Apis mellifera L., coletadas na Bahia. Bragantia. 2000;59(1):1-6, doi: 10.1590/S0006-87052000000100002.

18. Moreti ACCC, Arruda CMF, Marchini LC, Sodré GS. Análise polínica de amostras de méis de Apis mellifera L. (Hymenoptera, Apidae) da Chapada do Araripe, município de Santana do Cariri, Ceará, Brasil. Bol Ind Anim. 2005;62(3):235-244.

19. Sodré GS, Marchini LC, Carvalho CAL, Moreti ACCC. Pollen analysis in honey samples from the two main producing regions in the Brazilian northeast. Anais Acad Bras Ciências. 2007 Jan/Sep;79(3):381388, doi: 10.1590/S0001-37652007000300003.

20. Sodré GS, Marchini LC, Moreti ACCC, Carvalho CAL. Tipos polínicos encontrados em amostras de méis de Apis mellifera em Picos, Estado do Piauí. Ciên Rural. 2008 May/Jun;38(3):839-842, doi: 10.1590/S0103-84782008000300043.

21. Dórea MC, Novais JS, Santos FAR. Botanical profile of bee pollen from the southern coastal region of Bahia, Brazil. Acta Botanica Brasilica. 2010 Jul/Sep;24(3):862-867, doi: 10.1590/S010233062010000300028.

22. Oliveira PP, Berg CVD, Santos FAR. Pollen analysis of honeys from Caatinga vegetation of the State of Bahia, Brazil. Grana. 2010 Jun/Nov;49(1):66-75, doi: 10.1080/00173130903485122.

23. Costa SN, Alves RMO, Carvalho CAL, Conceição PJ. Fontes de pólen utilizadas por Apis mellifera Latreille na região semiárida. Ciên Anim Bras. 2015 Oct/Dec;16(4):491-49, doi: 10.1590/1089$6891 \mathrm{v} 16 \mathrm{i} 425538$.

24. Nascimento AS, Carvalho CAL, Sodré GS. The pollen spectrum of Apis mellifera honey from Reconcavo of Bahia, Brazil. J Sci Res \& Reports. 2015 Jan;6(6):426-438, doi: 10.9734/JSRR/2015/16799. 
25. Santos AMV, Nascimento AS, Santos JS, Silva SMPC, Lucas CIS, Carvalho CAL. Espectro polínico do mel de Apis mellifera L. coletado no período de produção melífera. Sci Electr Arch. 2019 Dec;12(6):6976, doi: 10.36560/1262019893.

26. Santos PC, Nascimento AS, Sodré GS, Carvalho CAL, Alves RMO, Ferreira MA, Andrade BR, Caldas MJM, Nunes LA, Estevinho LM. Pollen spectrum of honey of Apis mellifera L. and stingless bees (Hymenoptera: Apidae) from the semi-arid region of Bahia State, Brazil. Grana. 2020 Jul/May;59(5):377388, doi: $10.1080 / 00173134.2020 .1733074$.

27. Borges RLB, Lima LCL, Oliveira PP, Silva FHM, Novais JS, Dórea MC, Santos FAR. O pólen no mel do semi-árido brasileiro. In: Santos FAR, editor. Apium Plantae; Recife (PE): IMSEAR; 2006. p. 103118.

28. Lima LCL, Silva FHM, Araújo SS, Santos FAR. Morfologia polínica de espécies de Mimosa L. (Leguminosae) apícolas do semi-árido. In: Santos FAR, editor. Apium Plantae; Recife (PE): IMSEAR; 2006. p. 87-102.

29. SEI. Sistema de Informações Municipais. Superintendência de Estudos Econômicos e Sociais da Bahia (SEI) - 2018 [Internet]. [accessed 2018 May 15]. Available from: http://sim.sei.ba.gov.br

30. Louveaux J, Maurizio A, Vorwohl G. Methods of Melissopalynology. Bee World. 1978;59(4):139-157.

31. Iwama S, Melhem TS. The pollen spectrum of the honey of Tetragonisca angustula Latrelle (Apidae, Meliponinae). Apidologie. 1979;10(3):275-295, doi: 10.1051/apido:19790305.

32. Erdtman G. The acetolysis method. A revised description. Svensk Botanisk Tidskrift. 1960;54(4):561564.

33. Lima LCL, Silva FHM, Santos FAR. Palinologia de espécies de Mimosa L. (Leguminosae Mimosoideae) do semi-árido brasileiro. Acta Bot Bras. 2008;22(3):794-805, doi: 10.1590/S010233062008000300016

34. Buril MT, Santos FAR, Alves M. Diversidade polínica das Mimosoideae (Leguminosae) ocorrentes em uma área de Caatinga, Pernambuco, Brasil. Acta Bot Bras. 2010 Jan/Mar;24(1):53-64, doi: 10.1590/S0102-33062010000100006.

35. Silva CI, Ballesteros PLO, Palmero MA, Bauermann SG, Evaldt ACP, Oliveira PE. Catálogo polínico: palinologia aplicada em estudos de conservação de abelhas do gênero Xylocopa no Triângulo Mineiro. Uberlândia (MG): EDUFU; 2010. 154 p.

36. Silva FHM, Santos FAR, Lima LCL. Flora polínica das caatingas: Estação Biológica de Canudos (Canudos, Bahia, Brasil). Feira de Santana (BA): Mícron Bahia; 2016. 120 p.

37. Pielou EC. Ecological diversity. New York (US): J Wiley; 1975. 165 p.

38. Poole RW. An introduction to quantitative ecology. New York (US): McGraw-Hill; 1974. 480 p.

39. Oksanen J. Multivariate analyses of ecological communities in R: vegan tutorial - 2013 [Internet]. 2013 $\begin{array}{lllllll}\mathrm{Feb} & 8 & \text { [accessed } & 2020 & \mathrm{Jul} & 20]\end{array}$ http://www.pelagicos.net/MARS6910_spring2015/manuals/R_vegan_multivariate.pdf.

40. Hammer $\varnothing$, Harper DAT, Ryan PD. PAST: Paleontological statistics software package for education and data analysis. Palaeontol Electr. 2001;4(1):1-9.

41. Koppler K, Vororwoohl G, Koeniger N. Comparison of pollen spectra collected by four different subspecies of the honey bee Apis mellifera. Apidologie. 2007 Jul/Aug;38(4):341-353, doi: 10.1051/apido:2007020.

42. Aguiar CML, Zanella FCV, Martins CF, Carvalho CAL. Plantas visitadas por Centris spp. (Hymenoptera: Apidae) na Caatinga para obtenção de recursos florais. Neotr Entomol. 2003 Apr/Jun;32(2):247-259, doi: 10.1590/S1519-566X2003000200009.

43. Paula Neto FL, Almeida Neto RM. Apicultura nordestina: principais mercados, riscos e oportunidades. Fortaleza, Ceará: Banco do Nordeste do Brasil; 2006.78 p. (Série Documentos do ETENE; n. 12).

44. Demartelaere ACF, Oliveira AK, Góes GB, Lima GKL, Pereira MFS. A flora apícola no semi-árido brasileiro. Rev Verde. 2010 Jan/Mar;5(1):17-22, doi: 10.18378/rvads.v5i1.237.

45. Prado DE. As Caatingas da América do Sul. In: Leal IR, Tabarelli M, Silva JMC, editors. Ecologia e Conservação da Caatinga; Recife (PE): Ed. Universitária da UFPE; 2003. p. 3-35.

46. Cardoso DBOS, Queiroz LP. Diversidade de Leguminosae nas caatingas de Tucano, Bahia: implicações para a fitogeografia do semi-árido do Nordeste do Brasil. Rodriguésia. 2007 Aug/Mar;58(2):379-391, doi: 10.1590/2175-7860200758212.

47. Flora do Brasil 2020. Jardim Botânico do Rio de Janeiro - 2020 [Internet]. 2020 [accessed 2020 Jul 10]. Available from: http://floradobrasil.jbrj.gov.br/reflora/floradobrasil/FB115

48. Borges RLB, Jesus MC, Camargo RCR, Santos FAR. Pollen types in honey produced in caatinga vegetation, Brazil. Palynology. 2019 May;44(3):405-418, doi: 10.1080/01916122.2019.1617208.

49. Sodré GS. Características físico-químicas, microbiológicas e polínicas de amostras de méis de Apis mellifera L., 1758 (Hymenoptera: Apidae) dos estados do Ceará e Piauí [PhD thesis]. Piracicaba (SP): Universidade de São Paulo; 2005. 140 p. 
50. Santos FAR, Oliveira JM, Oliveira PP, Leite KRB, Carneiro CE. Plantas do semi-árido importantes para as abelhas. In: Santos FAR, editor. Apium Plantae; Recife (PE): IMSEAR; 2006. p. 5-130.

51. Nascimento AS, Carvalho CAL, Martins MLL. Plants visited by Apis mellifera L. (Hymenoptera: Apidae) in Recôncavo Baiano, State of Bahia, Brazil. Rev Agric. 2014;89(2):97-116, doi: 10.37856/bja.v89i2.123.

52. Vidal MG, Santana NS, Vidal D. Flora apícola e manejo de apiários na região do Recôncavo Sul da Bahia. Revista Acadêmica: Ciências Agrárias e Ambientais. 2008 Jun;6(4):503-509, doi: 10.7213/cienciaanimal.v6i4.11636.

53. Ramalho M, Kleinert-Giovannini A, Imperatriz-Fonseca VL. Important bee plants for stingless bees (Melipona and Trigonini) and Africanized honey-bees (Apis mellifera) in neotropical habitats: a review. Apidologie. $1990 \mathrm{Jul} /$ Aug;21(5):469-488.

54. Aguiar CML. Utilização de recursos florais por abelhas (Hymenoptera, Apoidae) em uma área de Caatinga (Itatim, Bahia, Brasil). Rev Bras Zool. 2003 Sep;20(3):457-467, doi: 10.1590/S010181752003000300015.

55. Pinheiro MP, Schlindwein C. Comunidade de abelhas (Hymenoptera, Apoidea) e plantas em uma área de Agreste pernambucano, Brasil. Rev Bras Entomol. 2008 Dec;52(4):625-636, doi: 10.1590/S008556262008000400014.

56. Oliveira PP. Análise palinológica de amostras de mel de Apis mellifera L. produzidas no estado da Bahia [PhD thesis]. Feira de Santana (BA): Universidade Estadual de Feira de Santa; 2009. 205 p.

57. Luz CFP, Thomé ML, Barth OM. Recursos tróficos de Apis mellifera L. (Hymenoptera, Apidae) na região de Morro Azul do Tinguá, Estado do Rio de Janeiro. Rev Bras Bot. 2007;30(1):29-36, doi: 10.1590/S0100-84042007000100004.

58. Locatelli E, Machado IC. Bee diversity and their floral resources in a fragment of a tropical altitudinal wet forest ("Brejos de altitude") in Northeastern Brazil. Acta Hortic. 2001 Nov;561:317-325, doi: 10.17660/ActaHortic.2001.561.48.

59. Marques LJP, Muniz FH, Lopes GS, Silva JM. Levantamento da flora apícola em Santa Luzia do Paruá, Sudoeste da Amazônia, Maranhão. Acta Bot Brasilica. 2011 Jan/Mar;20(1):141-149, doi: 10.1590/S010233062011000100017

60. Aires ERB, Freitas BM. Caracterização palinológica de algumas amostras de mel do estado do Ceará. Ciência Agron. 2001 Jan;32(1-2):22-28.

61. Santos RF, Kill LHP, Araújo JLP. Levantamento da flora melífera de interesse apícola no município de Petrolina-PE. Caatinga. $2006 \mathrm{Jul} / \mathrm{Sep}$;19(3):221-227.

62. Pereira FM, Freitas BM, Alves JE, Camargo RCR, Lopes MTR, Neto JMV, Rocha RS. Flora apícola no Nordeste. Teresina (PI): Embrapa Meio-Norte; 2004. 40 p. (Documentos; 104).

63. Machado IC, Lopes AV. (2006). Melitofilia em espécies de caatinga em Pernambuco e estudos relacionados existentes no ecossistema. In: Santos FAR, editor. Apium Plantae; Recife (PE): IMSEAR; 2006. p. 33-60.

64. Andrade JP, Costa SN, Santana ALA, Santos PC, Alves RMO, Carvalho CAL. Perfil polínico do mel de Melipona scutellaris Latreille, 1811 (Hymenoptera: Apidae) proveniente de colônias instaladas em área de agricultura familiar na Bahia. Rev Bras Agroecol. 2009 Nov;4(2):897-901.

65. Sekine LS, Toledo VAA, Caxambu MG, Chmura S, Takashiba EH, Sereia MJ, Marchini LC, Moreti ACCC. Melliferous flora and pollen characterization of honey samples of Apis mellifera L., 1758 in apiaries in the counties of Ubiratã and Nova Aurora, PR. Anais Acad Bras Ciências. 2013 Mar;85(1):307326, doi: 10.1590/S0001-37652013005000017. 